\title{
Use and implications of iron and other transition metals in environmental remediation processes
}

\author{
Khalil Hanna
}

Received: 27 July 2012 / Accepted: 27 August 2012 /Published online: 11 November 2012

(C) Springer-Verlag 2012

A large variety of contaminants are currently found in surface water, groundwater, and soil, including organic solvents, hydrocarbons and halogenated hydrocarbons, pesticides, pharmaceuticals, personal care products, and heavy metals. These contaminants become of special concern given their extensive use and the severe threats they cause to human health, aquatic life, and the environment. In light of this, a great deal of research has been performed in order to develop technologies capable of cleaning up contaminated matrices. In general, remediation technologies can be grouped into categories using thermal, physicochemical, chemical, or biological methods. Due to the complex nature of many contaminated systems, development of remediation methods that could clean all types of contaminants becomes a great challenge. The traditional technologies can be effective, but very often are costly and time-consuming. Thus, the removal of contaminants to a safe level, while at the same time rapidly, efficiently, and within a reasonable cost, is another challenge.

Fe and Mn oxides are generally the dominant redox-active components in soils, sediments, and other oxide-rich environments. These metal oxides can vary widely in physical and chemical characteristics and exist as micro- and nano-size particles. They affect a wide range of processes, including anaerobic degradation of organic matter, biogeochemical cycling and availability of trace elements, and the mobility, redox transformation and toxicity of organic and inorganic contaminants. Consequently, a range of environmental clean-

Responsible editor: Philippe Garrigues

K. Hanna $(\bowtie)$

École Nationale Supérieure de Chimie de Rennes, UMR CNRS

6226-Institut des Sciences Chimiques de Rennes-Equipe CIP,

Avenue du Général Leclerc, CS 50837,

35708 Rennes Cedex 7, France

e-mail: khalil.hanna@ensc-rennes.fr up technologies which use Fe or Mn chemistry to remediate contaminated waters or soils have been developed. From an engineering point of view, Fe- or Mn-mediated redox reactions can be potentially applicable for environmental remediation and protection. Iron is still the most important reactive material from both environmental and engineering points of view. Different forms of iron have been tested for activity against several different classes of environmental pollutants and for possible use as part of an environmental remediation technology. These have included microscale/nanoscale zerovalent metal particles, bimetallic reductive compounds, metal oxide or hydroxides, metal supported silica or pillared clays, and others. One of the most promising avenues of research involves the use of $\mathrm{Fe}^{0}$ - or $\mathrm{Fe}^{\mathrm{II}}$-based materials for the reductive transformation of halogenated compounds, nitro compounds, and oxyanions. Another engineering application is the use of Fe-based materials as catalysts for the chemical oxidation of recalcitrant pollutants via Fenton reactions. While not as extensively research, oxidative transformation of a variety of natural organics and xenobiotics by manganese (III/IV) oxides has been reported. Recognized as an important participant in environmental oxidation-reduction reactions, manganese(III/IV) oxides have significant potential for water and soil treatment application.

This special issue is not only a collection of key articles presented at the workshop "Iron and Environmental Chemistry" that was held in Nancy, France last November 2011 but also other research articles from contributed authors. The workshop aimed to chart the fundamental and applied aspects of iron species including structure, characterization, and surface reactions. It also aimed to outline the benefits and drawbacks of Fe-mediated redox reactions and their implications in environmental remediation processes.

In total, eight papers were accepted and included in this special issue. Most of the papers outline the use of iron in remediation processes via Fenton oxidation (modified 
Fenton or Fenton-like, heterogeneous Fenton, photoassisted Fenton, etc.). The main goal of these papers is to promote chemical oxidation reaction of target pollutants under soft conditions from both environmental and economic points of view (neutral $\mathrm{pH}$, low dose of catalyst, separation/recycling/reutilization of catalyst, low cost, etc.). The reaction, referred to as the Fenton reaction, is complex and capable of generating several kinds of radicals and higher oxidation states of the iron. While the conventional Fenton reaction is catalyzed by ferrous iron, the modified Fenton reaction (called also Fenton-like) uses ferric iron, zerovalent iron, or $\mathrm{Fe}$ oxides as the catalysts to degrade/transform hazardous compounds to less toxic forms. Photoassisted Fenton, electro-assisted Fenton, chelating agentenhanced Fenton, and Fe oxides-promoted heterogeneous Fenton-like reactions are subsequently developed to minimize some limitations of the conventional Fenton process. In two papers of this special issue, heterogeneous Fenton reactions using magnetite or goethite as iron source were shown to be efficient with respect to the treatment of contaminated waters and soils.

The work of Xiao and Lemley deals with the use of Mn (III/IV) cryptomelane-type manganese oxides for oxidative degradation of a fluoroquinolone antibiotic. Doping of the oxide with other metals such as molybdenum and vanadium proves effective for enhancing oxidative activity. Two papers deal with iron-induced transformation and photooxidation for contaminant removal (arsenic and octylphenol). The environmental reactions involving $\mathrm{Fe}$ or $\mathrm{Mn}$ reactive species are investigated for engineering application purposes. Two others papers analyze the links between the speciation of endogenous iron species and the leachability of metals in sediment. One of these papers presents both experimental and modeling studies to assess the mobilization of metals including iron from stabilized biotreated sediment. One paper deals with the use of a new magnetic coagulant
( $\mathrm{Fe}^{\mathrm{II}}-\mathrm{Fe}^{\mathrm{III}}$ oxide coated with fly ash) to investigate its coagulation-magnetic separation efficiency in freshwater containing harmful algal blooms.

This issue provides an overview of some of the different ways in which iron and other transition metals can be used in engineered systems. Overall, this special issue outlines both environmental and engineering aspects of the uses/ implications of $\mathrm{Fe}$ and $\mathrm{Mn}$ in remediation and fate of contaminants. The guest editor would like to thank all the authors for the high-quality research contributions to this special issue and also the reviewers who have critically evaluated the papers. I hope that this issue stimulates constructive discussions and innovative thinking that can lead to new insight and relevant applications. Finally, special thanks go to the Editor-in-Chief Philippe Garrigues and Editorial Assistant Emmanuelle Pignard-Péguet for their support and help.

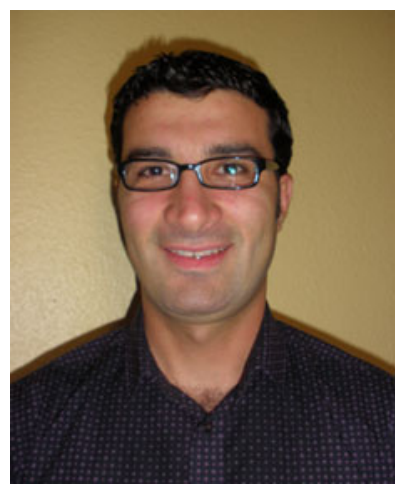

Khalil Hanna is currently a professor of Chemistry at the Graduate Engineering School of Chemistry of Rennes (France), one of the most prestigious institutes in Science and Engineering in France. He earned his $\mathrm{PhD}$ (2004) in Environmental Science and Technology from INSA of Lyon and joined the University of Nancy in 2005 as an associate professor. He has published over 60 peer-reviewed original research articles in top international journals and about 70 other contributions. He has been invited to give seminars at numerous prestigious universities and international conferences. His research interests focus on iron-mediated redox processes for soil and water treatments, environmental geochemistry, and modeling of contaminant transport in porous media. 